\title{
Effets du recépage sur les réserves glucidiques et lipidiques du «faux-vernis du Japon» (Ailanthus glandulosa Desf, Simarubacées) *
}

\author{
G Bory **, MD Sidibe, D Clair-Maczulajtys \\ Université Paris 7, groupe physiologie de l'arbre, 2 place Jussieu, 75005 Paris, France
}

(Reçu le 17 mai 1990; accepté le 18 octobre 1990)

\begin{abstract}
Résumé - Les jeunes plants de l'Ailanthus glandulosa sont remarquables par leur précocité à rejeter de souche et à drageonner.

L'enlèvement des parties aériennes provoque l'apparition de brins néoformés de diverses origines. Ils peuvent être issus de bourgeons préexistants dans l'hypocotyle, de bourgeons adventifs apparus sur la section de coupe et de bourgeons axillaires des cataphylles présentes sur la base des brins néoformés. Ces brins cataphyllaires s'affranchissent très facilement.

Les recépages successifs pratiqués en conditions contrôlées sur des plants de 8 mois provoquent globalement une stimulation de la production de matière sèche du compartiment racinaire. Ce traitement favorise les racines latérales plagiotropes par rapport au pivot.

Contrairement à certaines observations réalisées à propos d'autres feuillus, les recépages successifs ont un effet positif sur l'accumulation de réserves glucidiques dans le système souterrain. Ceci est mis en relation avec l'efficacité photosynthétique et les capacités de colonisation de cette essence.

Le compartimentage et la qualité des réserves glucidiques et lipidiques sont également modifiés sous l'effet de coupes répétées. Dans ce cas, les racines latérales vont constituer la zone principale d'accumulation de l'amidon, alors que le pivot joue ce rôle chez les plants témoins. La souche devient une zone d'accumulation de sucres solubles.

Pour les plants recépés 3 fois, la souche représente également une zone privilégiée d'accumulation de lipides. A $90 \mathrm{j}$, les brins développés après 3 coupes sont plus riches en lipides que les tiges des plants témoins. Le recépage provoque aussi une augmentation du degré d'insaturation des acides gras totaux. Ceci est particulièrement sensible dans les racines latérales et est dû à une plus grande importance des acides linoléique et linolénique. Après le recépage, le pivot, qui reste toujours pauvre en lipides, montre une prédominance des acides palmitique et stéarique.
\end{abstract}

Ailanthus glandulosa / compartimentage des réserves / recépage / rejet de souche / système racinaire

Summary - Effects of cutting back on the carbohydrate and lipid reserves in the tree of heaven (Ailanthus glandulosa Desf, Simaroubaceae). Seedlings of Ailanthus glandulosa show a remarkably early development of stump shoots and suckers.

The removal of aerial parts induced the development of shoots of different origins (fig 3). They may originate from pre-existing buds in the hypocotyl, adventitious buds on a cut section, or axillary buds

* Article présenté dans le cadre des activités du groupe d'étude de l'arbre.

** Correspondance et tirés à part 
of cataphylls present at the base of the new shoots. These cataphyllary shoots separated from seedlings very easily. After 2 or 3 cuts, seedlings also produced adventitious buds on roots and some started to develop suckers (tables I, II).

The 8-month-old seedlings raised under controlled greenhouse conditions were cut back 1, 2 or 3 times (fig 1). Dry weights and nutrient reserves of cut and intact seedlings were determined at 30-d intervals from the first cut.

The root system of the seedlings increased in dry weight throughout the successive cuts and the dry weight increase was higher in the lateral roots than in the taproot. At $90 d$, the largest increase in the total dry weight was observed in seedlings after 3 cuts. Growth of the collar which then became a stump only occurred in seedlings after 3 cuts (table III).

Contrary to some observations concerning deciduous trees, repetitive cutting back had a positive effect on the carbohydrate accumulation in the subterranean system. This result was related to the photosynthetic efficiency and the colonization of this species.

The distribution and the quality of the carbohydrate and lipid resenves were also modified by repetitive cuts. At 90 d, the carbohydrates were distributed in similar quantities between the aerial and subterranean parts of intact seedlings (table IV). After 2 or 3 successive cuts, the greater proportion of the starch reserve was then located in the root system. In this case, the lateral roots represented the principal site of starch accumulation whereas the taproot played this role in intact seedlings. The stump mainly accumulated soluble sugars (table IV).

In intact seedlings, the level of lipids was higher in the root system than in stems (table V). Cutting back induced a significant decrease in total lipids which occurred in shoots developed after 3 cuts at 30 and $90 \mathrm{~d}$. At the same time, the stump also represented the site of lipid accumulation for cut seedlings (table V). In other areas, cutting back caused an increase in the desaturation of the total fatty acids of the lateral roots, expressed as an increase in linoleic and linolenic acids (table V, fig 4). In contrast, the most important fatty acids in the taproot were palmitic and stearic acids (fig 4).

\section{Ailanthus glandulosa / reserve distribution / cutting back / stump shoots / root system}

\section{INTRODUCTION}

L'ailante glanduleux ou faux-vernis du Japon est une espèce arborescente pionnière remarquable par ses capacités à résister à différents types d'agressions. Elle se comporte d'ailleurs fort bien en milieu urbain. C'est un des arbres qui figure parmi les moins sensibles à la pollution (Kovacs et al, 1982). Ses particularités biochimiques lui permettent également de résister au développement de certains champignons parasites et de repousser les attaques des phytophages (Sussex et al, 1960; Ohmoto et al, 1976). Plusieurs expérimentations et observations ont montré que l'ailante évite et supporte une sécheresse prolongée (Dubroca et Bory, 1981), tolère des sols à haute teneur en chlorure de sodium et résiste à des baisses importantes de température (ClairMaczulajtys, 1984). Les explications de cette remarquable plasticité sont à recher- cher dans l'importance et la disponibilité des glucides racinaires, dans l'efficacité photosynthétique assurant un renouvellement rapide de ces réserves (Marek, 1988) et surtout dans l'aptitude pour cette espèce à développer des bourgeons adventifs. Ses potentialités organogènes (rhizogenèse adventive, bourgeonnement, mise à fleur) sont extrêmement précoces et augmentent les chances de survie des jeunes plants (Clair-Maczulajtys et Bory, 1983).

Cet article décrit l'effet du recépage sur les modifications de la quantité, de la qualité et du compartimentage des réserves glucidiques et lipidiques dans le système souterrain de l'ailante glanduleux. Ce travail complète les résultats déjà obtenus sur la résistance de cette espèce à différents types d'agressions (Dubroca et Bory, 1981; Bory, 1983; Clair-Maczulajtys, 1984). 


\section{MATÉRIELS ET MÉTHODES}

Les semis sont effectués en mars sous conditions contrôlées $\left(24 \pm 2{ }^{\circ} \mathrm{C}\right.$, éclairement de 100 W. $\mathrm{m}^{-2}$ en jours longs de $18 \mathrm{~h}$ ). Les jeunes plants se développent ainsi pendant 3 mois dans des bacs contenant un mélange de tourbe, de vermiculite et de terreau de feuilles en proportions équivalentes, sans apports nutritifs supplémentaires. Au début juin, ils sont transférés au jardin, dans leur bac d'origine. On les rentre en serre fin octobre lorsqu'ils ont perdu leurs feuilles; les conditions de culture sont similaires à celles utilisées pour la germination $\left(24 \pm 2{ }^{\circ} \mathrm{C}\right.$, jours longs de $18 \mathrm{~h}$, éclairement artificiel de 100 W. $\mathrm{m}^{-2}$ ). Le débourrement des bourgeons se produit $5 \mathrm{j}$ après. A ce moment, un $1^{\mathrm{er}}$ lot de plants est soumis à une ablation caulinaire, pratiquée dans la zone hypocotylaire à $1 \mathrm{~cm}$ audessus du collet. La distinction entre racine et tige est à cet endroit bien marquée par l'interruption du suber caulinaire (Clair-Maczulajtys, 1984). Sur un $2^{\mathrm{e}}$ lot, on pratique également un recépage dès l'entrée en şerre, puis $30 \mathrm{j}$ après on élimine de la même manière les brins qui se sont reformés. Enfin, sur un $3^{\theta}$ lot de plants, 3 coupes successives sont effectuées: la première au moment de l'entrée en serre, la deuxième après $30 \mathrm{j}$ de culture dans ces conditions et la dernière après $60 \mathrm{j}$. Tous ces lots de plants ainsi préparés sont élevés en serre pendant $90 \mathrm{j}$ sous les conditions d'environnement définies précédemment. Par ailleurs, on laisse se développer pendant la durée de l'expérience un lot de plants sur lequel aucun recépage n'est pratiqué. La figure 1 décrit ce dispositif expérimental.

Les observations morphologiques, les mesures et les prélèvements pour les dosages des métabolites glucidiques sont effectués tous les $30 \mathrm{j}$ après ce traitement. Pour les lipides totaux, il a été réalisé un dosage supplémentaire $5 \mathrm{j}$ après le dernier recépage. Les observations morphologiques ont été faites sur 20 plants. Pour les estimations des quantités de matière sèche ainsi que pour les dosages des métabolites, on donne les moyennes obtenues pour 5 individus. Les résultats relatifs à l'effet du nombre de recépages sur les quantités de matière sèche totale et de métabolites sont traités par l'analyse de variance $(P=0,05)$. Les effets significatifs des traitements sont caractérisés par la méthode des comparaisons multiples de Tukey.

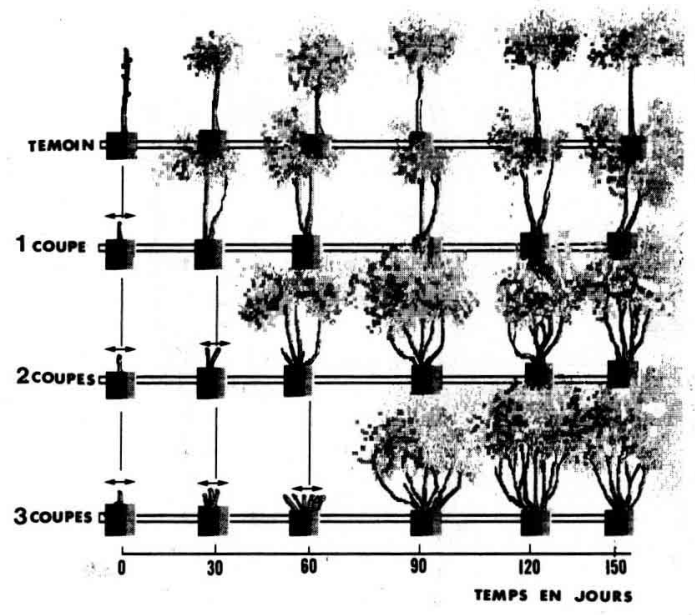

Fig 1. Protocole expérimental relatif aux recépages successifs. Les flèches doubles indiquent les périodes de coupe.

Les plants sont scindés en 4 parties morphologiquement distinctes. Il s'agit des tiges y compris les brins néoformés, des racines plagiotropes (racines latérales), du pivot et du collet ou de la souche. Pour le collet, chez les plants non recépés, on prélève une zone de jonction entre la tige et le pivot longue de $1 \mathrm{~cm}$ et englobant le collet proprement dit, bien visible chez cette espèce. Cette zone évoluera en souche avec le recépage et sera alors récoltée dans son intégrité (fig 2). La détermination de la matière sèche de ces différentes parties est effectuée par pesées après dessication à l'étuve à $105^{\circ} \mathrm{C}$.

Les prélèvements destinés aux dosages des glucides sont fixés immédiatement dans l'éthanol a $80 \%$. Les glucides sont extraits dans léthanol bouillant à $80 \%$ à partir de $2 \mathrm{~g}$ de matériel frais finement broyés. Après 3 extractions de $30 \mathrm{~min}$, les sucres solubles totaux sont dosés sur le filtrat par spectrophotométrie à $640 \mathrm{~nm}$ selon la méthode à l'anthrone décrite par Ashwell (1957). Le résidu des extractions précédentes est lavé 2 fois à l'éthanol $80 \%$, puis à l'eau distillée. Trois extractions à l'eau bouillante sont ensuite nécessaires pour extraire totalement l'amidon qui est, après hydrolyse à l'acide perchlorique, dosé selon le protocole de Macready et al (1950). 


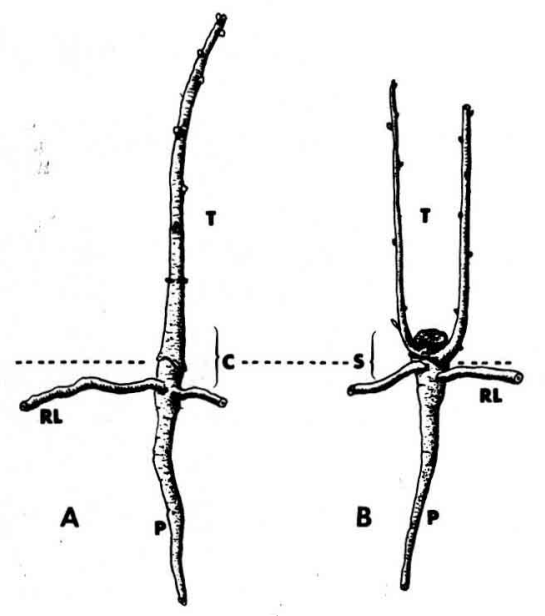

Fig 2. Localisation des prélèvements. A: plant non recépé, B: plant recépé $(x 0,6)$. (C: collet, $R L$, racines latérales ou plagiotropes, $P$ : pivot, S: souche, T: tiges).

Pour les lipides, $5 \mathrm{~g}$ de matériel frais sont fixés dans $50 \mathrm{ml}$ d'eau bouillante pendant $2 \mathrm{~min}$. On procède ensuite à l'extraction des lipides selon le protocole décrit précédemment (ClairMaczulajtys et Bory, 1987). La méthylation des acides gras totaux a été réalisée selon la méthode de Carreau et Dubacq (1978). L'analyse qualitative et quantitative des esters méthyliques est ensuite effectuée par chromatographie en phase gazeuse (appareil Varian-Aerograph couplé à un calculateur CDS 111). La température de la colonne est de $165^{\circ} \mathrm{C}$ et celle de l'injecteur de $220^{\circ} \mathrm{C}$.

\section{RÉSULTATS}

\section{Origine des pousses aériennes reconstituées}

A la suite de la $1^{\text {re }}$ coupe, des bourgeons adventifs se développent au niveau de la section de la tige sur un cal cicatriciel. D'autres se forment sur les flancs de la zone du collet; l'apparition de ces derniers est très rapide puisqu'ils deviennent visibles au bout de 5-6 j. Trente jours après 1 ou 2 recépages, il se forme toujours plus de bourgeons au niveau du collet que sur la section de la tige principale. C'est l'inverse pour les plants ayant subi 3 coupes (tableau I). Quel que soit le traitement, les bourgeons du collet n'apparaissent que pendant les 30 premiers jours tandis que ceux de la section de la tige principale

Tableau I. Nombre de bourgeons apparus 30 j et 90 j après la dernière coupe pratiquée. Les bourgeons apparus lors des coupes précédentes ne sont pas pris en compte, certains ont avorté, d'autres ont évolué en brins (moyenne sur 20 plants). L'écart type est donné entre parenthèses.

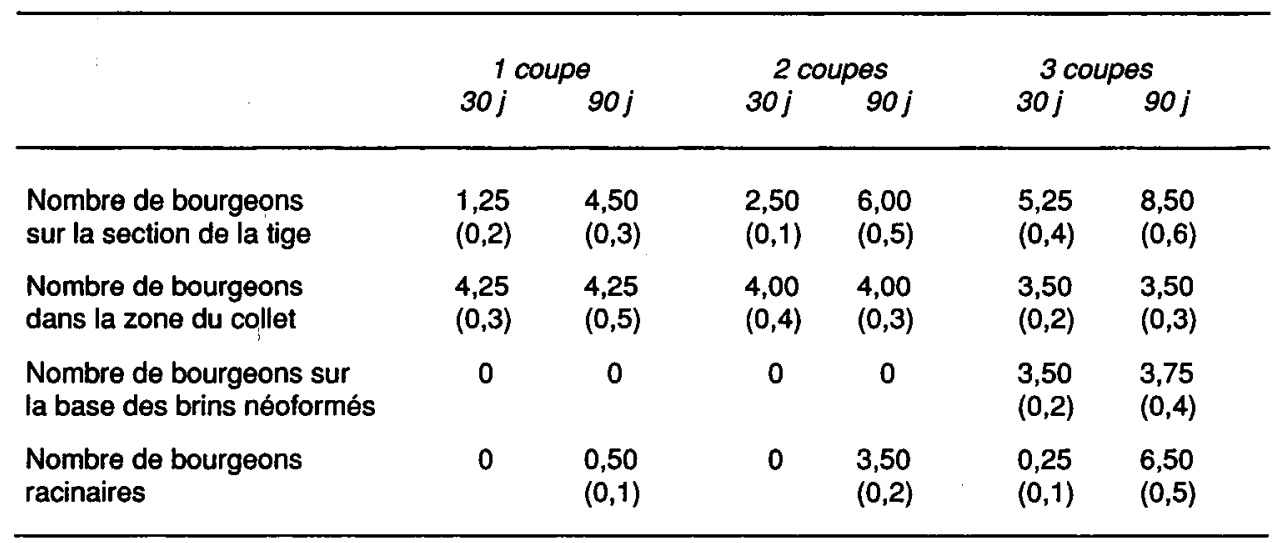


continuent de se former jusqu'à la fin de l'expérience (tableau I).

Dans le cas où le plant a subi 1 ou 2 coupes successives, il n'y a jamais de bourgeons visibles à l'œil nu sur la base des brins sectionnés. Au contraire, après 3 coupes, quelques-uns sont rapidement repérables (6-8 j après la dernière coupe). Iis correspondent au développement des bourgeons axillaires des premières formations foliaires (cataphylles). Ils donneront des brins à l'origine de la majorité des affranchissements observés à $90 \mathrm{j}$ (tableau II).

Une $4^{e}$ catégorie de bourgeons apparaît sur les plants recépés 2 ou 3 fois. II s'agit de bourgeons racinaires. Ceux-ci sont toujours beaucoup plus tardifs que les autres types de bourgeons et certains se développeront en drageons (tableau II).

Le nombre de brins sur le collet augmente progressivement avec les coupes successives. A $90 \mathrm{j}$ après le $3^{e}$ recépage, il $y$ a en moyenne 5 fois plus de brins développés qu'à la suite de la $1^{\text {re }}$ coupe (ta- bleau II). La figure 3 indique les différentes origines des tiges aériennes néoformées.

II faut souligner que les bourgeons adventifs apparus sur la section de la tige n'évoluent en axes feuillés que chez les jeunes plants à 3 coupes; plus de la moitié d'entre eux disparaîtra pendant la durée de l'expérience. Dans ce cas également, les brins ayant pour origine les bourgeons axillaires des cataphylles sont nombreux à s'affranchir (environ $\mathbf{8 0 \%}$ d'entre eux). Les

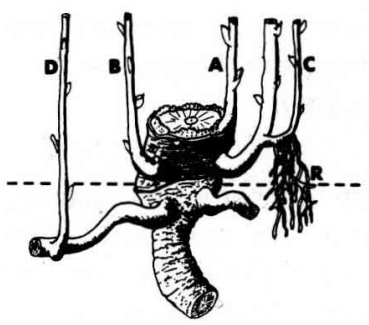

Fig 3. Différents types de rejets $(x 1,2), A$ : brins issus de bourgeons latents, $B$ : bourgeons adventifs, C: brins "cataphyllaires", D: drageons, $\mathrm{R}$ : racines adventives).

Tableau II. Nombre de brins présents à $30 \mathrm{j}$ et à $90 \mathrm{j}$ après la dernière coupe. II s'agit des pousses de $1 \mathrm{~cm}$; les mesures correspondent à des moyennes effectuées sur 20 individus, l'écart type est donné entre parenthèses.

\begin{tabular}{|c|c|c|c|c|c|c|}
\hline & \multicolumn{2}{|c|}{1 coupe } & \multicolumn{2}{|c|}{2 coupes } & \multicolumn{2}{|c|}{3 coupes } \\
\hline & $30 j$ & $90 j$ & $30 j$ & $90 j$ & $30 j$ & $90 j$ \\
\hline $\begin{array}{l}\text { Nombre de brins } \\
\text { sur la section de tige }\end{array}$ & 0 & 0 & 0 & 0 & $\begin{array}{l}6,50 \\
(1,2)\end{array}$ & $\begin{array}{l}3,00 \\
(0,5)\end{array}$ \\
\hline $\begin{array}{l}\text { Nombre de brins } \\
\text { au niveau du collet }\end{array}$ & $\begin{array}{l}1,25 \\
(0,7)\end{array}$ & $\begin{array}{l}1,50 \\
(0,8)\end{array}$ & $\begin{array}{l}2,25 \\
(0,5)\end{array}$ & $\begin{array}{l}2,00 \\
(0,5)\end{array}$ & $\begin{array}{l}5,00 \\
(0,4)\end{array}$ & $\begin{array}{l}6,75 \\
(0,5)\end{array}$ \\
\hline Nombre de drageons & 0 & 0 & 0 & $\begin{array}{l}1,25 \\
(0,7)\end{array}$ & 0 & $\begin{array}{l}1,25 \\
(0,1)\end{array}$ \\
\hline $\begin{array}{l}\text { Nombre de brins } \\
\text { cataphyllaires }\end{array}$ & 0 & 0 & 0 & $\begin{array}{l}1,00 \\
(0,3)\end{array}$ & 0 & $\begin{array}{l}1,25 \\
(0,4)\end{array}$ \\
\hline $\begin{array}{l}\text { Nombre de brins } \\
\text { affranchis }\end{array}$ & 0 & 0 & 0 & 0 & 0 & $\begin{array}{l}1,25 \\
(0,8)\end{array}$ \\
\hline
\end{tabular}


autres brins capables de former leur propre système racinaire sont situés à la base du collet.

Le nombre de drageons ne paraît pas être en relation avec l'intensité du recépage. Cependant, il ne s'en développe pas après 1 seule coupe (tableau II). Leur développement est toujours tardif. L'émergence des tiges aériennes ne se produit qu'à partir de $60 \mathrm{j}$ après la dernière coupe et ceci quel que soit le traitement.

\section{Évolution du poids de la matière sèche en fonction de la fréquence des recépages}

En ce qui concerne les plants entiers, après $60 \mathrm{j}$, on constate qu'une seule coupe entraîne par rapport au témoin, une baisse significative du poids de la matière sèche. Au contraire, les plus fortes valeurs de matière sèche totale par rapport au témoin sont observées après 3 recépages successifs et à $90 \mathrm{j}$ (tableau III).

Pour le compartiment racinaire, après 1,2 ou 3 coupes, c'est surtout la matière sèche des racines latérales qui augmente significativement; elle représente alors 2-3 fois celle du pivot. Au contraire, les plants qui n'ont jamais été sectionnés ont un pivot dont le poids de matière sèche croît régulièrement (tableau III).

A 30,60 ou $90 \mathrm{j}$, le nombre de recépages induit de façon significative une augmentation du poids de matière sèche du collet. A la fin de l'expérience cette zone particulière devient une véritable souche qui représente près de $39 \%$ du poids total du plant (tableau III).

Après $90 \mathrm{j}$, les brins néoformés représentent respectivement pour 1,2 et 3 coupes $7,76 \mathrm{~g}, 6,42 \mathrm{~g}$, et $5,3 \mathrm{~g}$ soit $46 \%$, $37 \%$ et $22 \%$ de la matière sèche totale du plant. Les recépages successifs sont sans
Tableau III. Évolution du poids de matière sèche (en g) après une coupe (1C), 2 coupes successives (2C), 3 coupes successives (3C) et chez le plant témoin $(\mathrm{OC})$. Les valeurs suivies de la même lettre ne sont pas significativement différentes au seuil $P \leq 0,05$ selon le test de Tukey.

\begin{tabular}{|c|c|c|c|c|}
\hline & \multicolumn{4}{|c|}{ Temps en jours } \\
\hline & 0 & 30 & 60 & 90 \\
\hline \multicolumn{5}{|l|}{ Tiges ou brins } \\
\hline $\mathrm{OC}$ & 1,63 & $3,24^{a}$ & $6,61^{a}$ & $6,48^{a}$ \\
\hline $1 \mathrm{C}$ & - & $1,01^{b}$ & $1,24^{b}$ & $7,76^{a}$ \\
\hline $2 \mathrm{C}$ & - & $1,46^{b}$ & $3,68^{c}$ & $6,42^{a}$ \\
\hline & & $1,87^{b}$ & $2,95^{c}$ & $5,35^{a}$ \\
\hline \multicolumn{5}{|c|}{ Collet ou souche } \\
\hline OC & $0,82^{a}$ & $0,90^{a}$ & $1,11^{a}$ & $1,21 a$ \\
\hline $1 \mathrm{C}$ & $0,82^{a}$ & $1,41^{b}$ & $1,50^{a}$ & $1,61^{b}$ \\
\hline $2 \mathrm{C}$ & $1,41^{b}$ & $2,43^{c}$ & $2,80^{b}$ & $3,90^{c}$ \\
\hline $3 C$ & $2,43^{c}$ & $5,12^{d}$ & $7,48^{c}$ & $9,42^{d}$ \\
\hline \multicolumn{5}{|l|}{ Pivot } \\
\hline $\mathrm{OC}$ & $1,29^{a}$ & $1,92^{a}$ & $3,30^{\mathrm{a}}$ & $3,72^{a}$ \\
\hline $1 \mathrm{C}$ & $1,29^{a}$ & $1,31^{a}$ & $1,89 b c$ & $1,91^{b c}$ \\
\hline $2 \mathrm{C}$ & $1,31^{a}$ & $1,44^{a}$ & $1,52^{b}$ & $1,75^{b}$ \\
\hline $3 C$ & $1,44^{a}$ & $1,59^{a}$ & $2,01^{c}$ & $2,55^{\mathrm{C}}$ \\
\hline \multicolumn{5}{|c|}{ Racines latérales } \\
\hline $\mathrm{OC}$ & $0,90^{a}$ & $1,46^{\mathrm{a}}$ & $1,87 a$ & $1,89^{\mathrm{a}}$ \\
\hline $1 C$ & $0,90^{a}$ & $3,22^{b}$ & $3,95^{b}$ & $5,67^{b}$ \\
\hline $2 C$ & $3,22^{b}$ & $4,12^{c}$ & $4,96^{c}$ & $5,20^{\circ}$ \\
\hline $3 C$ & $4,12^{c}$ & $5,36^{d}$ & $6,42^{\mathrm{d}}$ & $7,38^{c}$ \\
\hline \multicolumn{5}{|l|}{ Plant entier } \\
\hline $\mathrm{OC}$ & $4,64^{a}$ & $7,52^{\mathrm{a}}$ & $12,89^{a}$ & $13,30^{a}$ \\
\hline $1 \mathrm{C}$ & $3,01^{b}$ & $6,95^{a}$ & $8,58^{b}$ & $16,95^{\mathrm{ab}}$ \\
\hline $2 \mathrm{C}$ & $5,94^{c}$ & $9,45^{b}$ & $12,96^{\mathrm{a}}$ & $17,27^{b}$ \\
\hline $3 C$ & $7,99^{d}$ & $13,94^{c}$ & $18,86^{c}$ & $24,70^{\mathrm{c}}$ \\
\hline
\end{tabular}

effet sur l'augmentation de la matière sèche des tiges. Ils favorisent donc l'accroissement des parties souterraines par rapport au développement des brins et d'une manière générale augmentent le poids total du plant (tableau III).

A la fin de l'expérience, les plants témoins montrent une distribution de la ma- 
tière sèche équivalente entre les parties aériennes (A) et souterraines (S). Ce rapport A/S diminue avec l'augmentation du nombre de coupes, marquant ainsi l'importance du développement du système souterrain par rapport aux brins néoformés (tableau (II).

\section{Évolution des quantités de glucides et de lipides}

Par rapport au plant témoin, on relèvera, pour la plante entière et après 3 recépages, une accumulation significative de sucres solubles et d'amidon à la fin de l'expérience. A ce moment, la stimulation de l'accumulation d'amidon apparaît plus forte après 1 seule coupe. Elle est due à une augmentation très importante de l'amidon dans les tiges. Ces dernières représentent alors un site important de rétention de glucides, alors que chez le plant témoin, ils se répartissent en quantités comparables dans les parties aériennes et souterraines (tableau IV). Au contraire, pour les plants ayant été recépés 2 ou 3 fois, la majorité des réserves amylacées se trouve à la fin de l'expérience dans le système racinaire. Dans ce cas, les racines latérales vont constituer la zone principale d'accumulation de l'amidon alors que le pivot s'appauvrit en métabolites glucidiques de façon significative. Chez le témoin non recépé, il demeure avec les tiges un site important de stockage de l'amidon et des sucres solubles alors que les racines latérales sont remarquables par leur pauvreté en amidon (tableau IV).

Les coupes successives ont un effet stimulant sur l'accumulation de sucres solubles dans la souche qui devient, après 3 recépages et à $90 \mathrm{j}$, un véritable réservoir à sucres solubles pour l'ensemble du plant (tableau IV).
Les plants ayant été recépés 1 fois sont à $90 \mathrm{j}$ bien moins riches en lipides que les plants témoins. En particulier, dans le pivot, on note une diminution significative des lipides totaux. II en est de même pour les plants ayant subi 2 ou 3 coupes successives. Seuls, les brins néoformés après 3 coupes sont plus riches en lipides que la tige des plants témoins. Les recépages successifs entraînent à $90 \mathrm{j}$ une augmentation significative des lipides totaux dans les nouveaux brins (tableau V).

La souche constitue également, à $90 \mathrm{j}$, pour les plants recépés 3 fois, une importante zone d'accumulation de lipides. Cinq jours après la dernière coupe, les quantités de lipides présentes dans la souche ou dans les racines latérales sont nettement plus élevées pour les plants coupés 2 ou 3 fois (tableau V).

L'analyse de la composition en acides gras des lipides totaux fait apparaître que 2 ou 3 recépages successifs provoquent dès 5 j une augmentation du taux d'insaturation, spécialement dans les racines latérales. Ce niveau élevé du degré d'insaturation se maintient jusqu'à $90 \mathrm{j}$ (tableau V). Ce phénomène est alors dû, comme le mentionne la figure 4 , à une forte proportion des acides linoléique $\left(\mathrm{C}_{18: 2}\right)$ et linolénique $\left(C_{18: 3}\right)$. Au contraire, dans les brins néoformés, 3 coupes successives provoquent une diminution du taux d'insaturation des acides gras (tableau V). Ceci est à mettre en relation avec l'augmentation de l'importance du $\mathrm{C}_{18: 0}$ (acide stéarique) et du $\mathrm{C}_{16: 0}$ (acide palmitique) et avec la diminution de l'acide linoléique (fig 4).

Chez le témoin, ainsi que chez les plants recépés, le taux d'insaturation des acides gras est généralement plus bas dans le pivot que dans les autres compartiments. Les coupes successives accentuent cette particularité (tableau V). D'une manière générale, la composition en 
Tableau IV. Évolution (en fonction du temps écoulé après la dernière coupe) des quantités de glucides (amidon et sucres solubles) dans les différentes parties de plants d'ailante récépés. Les quantités sont exprimées en g. $0 \mathrm{C}$ : témoin non recépé, $1 \mathrm{C}:$ une coupe, $2 \mathrm{C}: 2$ coupes, $3 \mathrm{C}: 3$ coupes. Les valeurs suivies de la même lettre ne sont pas significativement différentes au seuil $P \leq 0,05$ selon le test de Tukey.

\begin{tabular}{|c|c|c|c|c|c|c|c|c|}
\hline & 0 & $\begin{array}{l}30 \\
\text { Sucres }\end{array}$ & $\begin{array}{c}60 \\
\text { solubles }\end{array}$ & $90^{\text {Temp }}$ & urs 0 & ${ }^{30}$ Ami & don & 90 \\
\hline \multicolumn{9}{|l|}{ Tiges } \\
\hline OC & 0,132 & $0,460^{a}$ & $0,567^{a}$ & $0,435^{a}$ & 0,015 & $0,593^{a}$ & $0,568^{a}$ & $0,598^{a}$ \\
\hline $1 \mathrm{C}$ & - & $0,088^{b}$ & $0,121^{b}$ & $0,603^{b}$ & - & $0,301^{b}$ & $0,335^{b}$ & $2,309^{b}$ \\
\hline $2 C$ & - & $0,128^{c}$ & $0,166^{b}$ & $0,512^{b c}$ & - & $0,356^{b}$ & $0,958^{c}$ & $0,567^{a}$ \\
\hline $3 C$ & $\rightarrow$ & $0,164^{c}$ & $0,245^{c}$ & $0,478^{a c}$ & - & $0,195^{c}$ & $0,222^{d}$ & $0,374^{c}$ \\
\hline \multicolumn{9}{|l|}{ Collet souche } \\
\hline OC & $0,156^{a}$ & $0,099^{a}$ & $0,180^{a}$ & $0,071^{a}$ & $0,000^{a}$ & $0,024^{a}$ & $0,186^{a}$ & $0,119^{a}$ \\
\hline $1 \mathrm{C}$ & $0,156^{a}$ & $0,203^{b}$ & $0,155^{a}$ & $0,089 a$ & $0,000^{a}$ & $0,470^{b}$ & $0,260^{a}$ & $0,057^{b}$ \\
\hline $2 \mathrm{C}$ & $0,203^{a}$ & $0,187^{b}$ & $0,132^{a}$ & $0,518^{b}$ & $0,470^{b}$ & $0,661^{c}$ & $0,215^{a}$ & $0,180^{c}$ \\
\hline $3 C$ & $0,187 a$ & $0,236^{b}$ & $0,812^{b}$ & $1,328^{c}$ & $0,661^{c}$ & $0,088^{a}$ & $0,161^{a}$ & $0,183^{c}$ \\
\hline \multicolumn{9}{|l|}{ Pivot } \\
\hline $\mathrm{OC}$ & $0,201^{a}$ & $0,209^{a}$ & $0,185^{a}$ & $0,222^{a}$ & $0,142^{a}$ & $0,202^{a}$ & $0,366^{a}$ & $0,455^{a}$ \\
\hline $1 \mathrm{C}$ & $0,201^{a}$ & $0,354^{b}$ & $0,441^{b}$ & $0,116^{b}$ & $0,142^{a}$ & $0,281^{b}$ & $0,302^{a}$ & $0,189^{b}$ \\
\hline $2 \mathrm{C}$ & $0,354^{b}$ & $0,131^{c}$ & $0,067^{c}$ & $0,038^{c}$ & $\cdot 0,281^{b}$ & $0,185^{a}$ & $0,123^{b}$ & $0,130^{\mathrm{b}}$ \\
\hline $3 C$ & $0,131^{c}$ & $0,076^{d}$ & $0,069 c$ & $0,031^{c}$ & $0,185^{a}$ & $0,020^{c}$ & $0,049 c$ & $0,050^{c}$ \\
\hline \multicolumn{9}{|c|}{ Racines latérales } \\
\hline$O C$ & $0,073^{a}$ & $0,213^{a}$ & $0,123^{a}$ & $0,182^{a}$ & $0,102^{a}$ & $0,118^{a}$ & $0,148^{a}$ & $0,027^{a}$ \\
\hline $1 \mathrm{C}$ & $0,073^{a}$ & $0,236^{a}$ & $0,452^{b}$ & $0,268^{a}$ & $0,102^{a}$ & $0,437^{b}$ & $0,329 b$ & $1,092^{b}$ \\
\hline $2 C$ & $0,236^{b}$ & $0,387^{b}$ & $0,292^{\mathrm{c}}$ & $0,361^{b}$ & $0,437^{b}$ & $1,168^{c}$ & $1,114^{c}$ & $1,305^{c}$ \\
\hline $3 \mathrm{C}$ & $0,387^{b}$ & $0,488^{b}$ & $0,496^{b}$ & $0,517^{c}$ & $1,168 \mathrm{c}$ & $1,612^{d}$ & $1,884^{d}$ & $2,215^{d}$ \\
\hline \multicolumn{9}{|l|}{ Plant entier } \\
\hline OC & $0,381^{a}$ & $0,981^{a}$ & $1,491^{a}$ & $0,911^{a}$ & $0,259^{a}$ & $0,937^{a}$ & $1,269^{a}$ & $1,199^{a}$ \\
\hline $1 \mathrm{C}$ & $0,249^{a}$ & $1,002^{a}$ & $1,168^{b}$ & $1,076^{a}$ & $0,244^{a}$ & $1,489^{b}$ & $1,227^{a}$ & $3,646^{b}$ \\
\hline $2 C$ & $0,793^{b}$ & $0,833^{a}$ & $0,656^{c}$ & $1,428^{b}$ & $1,188^{b}$ & $2,371^{c}$ & $2,410^{b}$ & $2,183^{c}$ \\
\hline $3 C$ & $0,705^{b}$ & $0,965^{a}$ & $1,622^{a}$ & $2,354^{c}$ & $2,015^{\mathrm{c}}$ & $1,912^{c}$ & $2,316^{b}$ & $2,822^{\circ}$ \\
\hline
\end{tabular}

acides gras du pivot des plants recépés est dominée par la présence des acides palmitique $\left(\mathrm{C}_{16: 0}\right)$, stéarique $\left(\mathrm{C}_{18: 0}\right)$. Chez les plants non recépés, l'acide oléique $\left(C_{18: 1}\right)$ est mieux représenté, mais son importance décroît avec les recépages successifs (fig 4). Sa composition en acides gras est tout à fait comparable à celle des racines latérales. Chez ces dernières, à $90 \mathrm{j}$, les acides linoléique $\left(\mathrm{C}_{18: 2}\right)$ et linolénique $\left(\mathrm{C}_{18: 3}\right)$ représentent les acides gras majeurs. Contrairement au pivot, les recépages successifs induisent une diminution de l'acide palmitique $\left(\mathrm{C}_{16: 0}\right)$. 


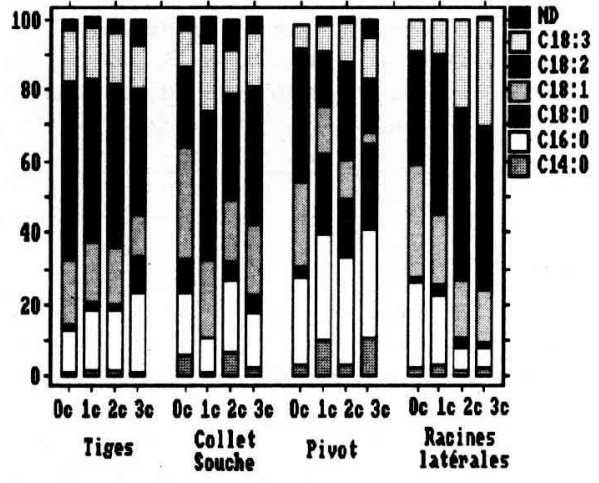

Fig 4. Évolution de la composition en acides gras ( $\%$ des acides gras totaux) des différents compartiments étudiés (après $90 \mathrm{j}$ ). 0c: témoin, $1 \mathrm{c}$ : une coupe, 2c: 2 coupes, $3 c$ : 3 coupes. ND: fraction non déterminée.

\section{DISCUSSION}

L'ailante est donc une espèce particulièrement intéressante pour ses capacités à résister à des coupes successives. En effet, 3 conditions sont réunies :

- il existe un stock de bourgeons capables de se développer en rejets,

- cette espèce possède des capacités organogènes exceptionnelles permettant en particulier un renouvellement des bourgeons adventifs,

- l'ailante possède la majorité de ses réserves énergétiques dans le système souterrain (Clair-Maczulajtys, 1984).

Les jeunes plants d'ailante recépés sont remarquables par leur précocité à rejeter de souche et à drageonner. En fait, des potentialités de régénération exceptionnelles ont été décrites chez des germinations de $15 \mathrm{j}$ (Clair-Maczulajtys et Bory, 1983). L'hypocotyle se révèle déjà une zone organogène très importante, capable de s'enraciner ou de former naturellement des bourgeons adventifs dans le parenchyme cortical. Des organes plus âgés comme des segments de tige internodaux sont également capables de produire des bourgeons adventifs (Caruso, 1974). Au niveau du collet, les bourgeons qui se développent en brins après la coupe sont issus de petits massifs méristématiques indifférenciés préexistants. La coupe est donc à l'origine de leur évolution en bourgeons puis en tiges, mais non responsable de leur démarrage (Clair-Maczulajtys, 1984). Cependant, en l'absence de données sur la quantité de ces massifs précurseurs, rien n'interdit de penser que la coupe pourrait aussi stimuler leur formation. Les brins qui se développent sur la souche ne sont donc jamais issus de bourgeons axillaires latents. Au contraire, chez d'autres espèces comme Betula pendula et $B$ pubescens, les brins se forment à partir des bourgeons axillaires latents et les brins d'origine adventive ne se développent que lorsque les plants sont incapables de rejeter à partir des bourgeons dormants (Rinne et al, 1987). Ici, les brins apparaissent très tardivement sur le cal cicatriciel de la tige et toujours après 3 coupes successives. La formation des brins issus de zones méristématiques préexistantes semble donc être privilégiée au départ. Les coupes successives entraînent une augmentation du nombre de pousses par souche. Ceci a été signalé chez d'autres espèces capables de supporter ce traitement (Kauppi et al, 1988). Les auteurs mentionnent que Betula pubescens est avantagée à la fois par une réserve considérable de bourgeons latents et par une capacité à en reformer.

Les drageons ne se développent qu'après 3 enlèvements successifs des brins. II faut signaler que la seule ablation du bourgeon terminal de la tige en croissance provoque la rentrée en activité des bourgeons racinaires chez des plants de 2 ans; la section de la tige au niveau du collet n'en produisant pas (Clair- 
Tableau V. Évolution des quantités (en $\mathrm{mg}$ ) de lipides totaux saponifiables (T) et du degré d'insaturation (I) des différents compartiments étudiés. $O \mathrm{C}$ : témoin non recépé, $1 \mathrm{C}:$ une coupe, $2 \mathrm{C}: 2$ coupes, $3 C: 3$ coupes. Pour chaque partie de la plante, les valeurs suivies de la même lettre ne sont pas significativement différentes, $P \leq 0,05$ selon le test de Tukey.

\begin{tabular}{|c|c|c|c|c|c|c|c|c|}
\hline & \multicolumn{8}{|c|}{ Temps en jours } \\
\hline & \multicolumn{2}{|c|}{0} & \multicolumn{2}{|r|}{5} & \multicolumn{2}{|c|}{30} & \multicolumn{2}{|c|}{90} \\
\hline & $T$ & 1 & $T$ & 1 & $T$ & 1 & $T$ & I \\
\hline \multicolumn{9}{|c|}{ Tiges ou brins } \\
\hline$O C$ & 8,1 & $(77,0)$ & 7,3 & $(76,4)$ & $16,2^{a}$ & $(78,1)$ & $64,3^{a}$ & $(79,3)$ \\
\hline $1 C$ & - & & - & - & $12,0^{\mathrm{a}}$ & $(83,3)$ & $38,0^{\mathrm{b}}$ & $(79,8)$ \\
\hline $2 C$ & - & & - & - & $16,8^{a}$ & $(76,1)$ & $79,0^{\mathrm{C}}$ & $(79,3)$ \\
\hline $3 C$ & - & & - & - & $26,2^{b}$ & $(59,8)$ & $92,6^{d}$ & $(65,4)$ \\
\hline \multicolumn{9}{|c|}{ Collet ou souche } \\
\hline $\mathrm{OC}$ & $2,3^{a}$ & $(78,4)$ & $2,5^{a}$ & $(80,1)$ & $4,0^{\mathrm{a}}$ & $(79,4)$ & $15,0^{\mathrm{a}}$ & $(77,5)$ \\
\hline $1 \mathrm{C}$ & $2,3^{a}$ & $(78,4)$ & $1,7^{a}$ & $(89,8)$ & $4,2^{a}$ & $(80,0)$ & $4,0^{b}$ & $(89,0)$ \\
\hline $2 \mathrm{C}$ & $4,2^{b}$ & $(80,0)$ & $11,6^{\mathrm{b}}$ & $(89,6)$ & $7,1^{\mathrm{b}}$ & $(72,8)$ & $19,9^{a}$ & $(68,4)$ \\
\hline $3 C$ & $7,1^{c}$ & $(72,8)$ & $31,3^{c}$ & $(89,0)$ & $15,9^{c}$ & $(71,9)$ & $89,5^{c}$ & $(77,3)$ \\
\hline \multicolumn{9}{|l|}{ Pivot } \\
\hline$O C$ & $2,8^{a}$ & $(68,7)$ & $3,2^{\mathrm{a}}$ & $(69,9)$ & $4,0^{a}$ & $(68,5)$ & $53,6^{a}$ & $(69,1)$ \\
\hline $1 \mathrm{C}$ & $2,8^{a}$ & $(68,7)$ & $3,9^{a}$ & $(46,5)$ & $3,+1 b$ & $(46,3)$ & $5,3^{b}$ & $(38,2)$ \\
\hline $2 \mathrm{C}$ & $3,1^{\mathrm{a}}$ & $(46,3)$ & $4,6^{b}$ & $(37,9)$ & $2,6^{b}$ & $(46,4)$ & $3,7^{b}$ & $(49,7)$ \\
\hline $3 \mathrm{C}$ & $2,6^{a}$ & $(46,4)$ & $4,9^{b}$ & $(33,7)$ & $2,9^{b}$ & $(30,6)$ & $6,3^{b}$ & $(33,7)$ \\
\hline \multicolumn{9}{|c|}{ Racines latérales } \\
\hline OC & $2,2^{a}$ & $(72,2)$ & $3,4^{a}$ & $(71,3)$ & $4,5^{a}$ & $(72,5)$ & $23,2^{a}$ & $(74,3)$ \\
\hline $1 \mathrm{C}$ & $2,2^{a}$ & $(72,2)$ & $5,0^{\mathrm{b}}$ & $(89,7)$ & $10,0^{b}$ & $(74,7)$ & $17,0^{\mathrm{b}}$ & $(74,4)$ \\
\hline $2 \mathrm{C}$ & $10,0^{b}$ & $(74,7)$ & $34,4^{c}$ & $(89,2)$ & $12,4^{b}$ & $(89,2)$ & $13,5^{c}$ & $(89,1)$ \\
\hline $3 C$ & $12,4^{b}$ & $(89,2)$ & $51,5^{d}$ & $(90,7)$ & $22,5^{c}$ & $(89,1)$ & $14,8^{\mathrm{c}}$ & $(90,5)$ \\
\hline \multicolumn{9}{|l|}{ Plant entier } \\
\hline $\mathrm{OC}$ & \multicolumn{2}{|c|}{$15,5^{a}$} & \multicolumn{2}{|c|}{$16,5^{a}$} & \multicolumn{2}{|c|}{$28,7^{a}$} & \multicolumn{2}{|c|}{$156,1^{a}$} \\
\hline $1 \mathrm{C}$ & \multicolumn{2}{|c|}{$7,4^{b}$} & \multicolumn{2}{|c|}{$10,6^{b}$} & \multicolumn{2}{|c|}{$29,3^{a}$} & \multicolumn{2}{|c|}{$64,3^{b}$} \\
\hline $2 \mathrm{C}$ & \multicolumn{2}{|c|}{$17,3^{a}$} & \multicolumn{2}{|c|}{$50,6^{c}$} & \multicolumn{2}{|c|}{$38,8^{b}$} & \multicolumn{2}{|c|}{$116,1^{\mathrm{c}}$} \\
\hline $3 \mathrm{C}$ & \multicolumn{2}{|c|}{$22,0^{\circ} \mathrm{C}$} & \multicolumn{2}{|c|}{$87,7^{d}$} & \multicolumn{2}{|c|}{$67,5^{c}$} & \multicolumn{2}{|c|}{$203,2^{d}$} \\
\hline
\end{tabular}

Maczulajtys, 1984). Depuis les travaux d'Eliasson (1971) et de Schier (1973), it est bien évident que la formation des drageons est régulée par la dominance apicale. Les réserves glucidiques ne paraissent pas jouer un rôle direct dans la levée d'inhibition des bourgeons racinaires (Tew, 1970) ou des bourgeons latents des souches (Vogt et Cox, 1970). Par contre, les réserves des racines sont largement utilisées dans la croissance des brins pendant leur phase hétérotrophe de développement (Bamber et Humphreys, 1965; Schier et Zasada, 1973; Taylor et al, 1982). L'importance des glucides racinaires chez l'ailante est une des explica- 
tions à ses capacités colonisatrices par drageonnement (Clair-Maczulajtys, 1984).

La taille et la défoliation entraînent le plus souvent une hydrolyse et une diminution de réserves, même après reconstitution du feuillage (Abusrewill et al, 1983; Reich, 1985; Parker, 1979). Chez des jeunes plants de Betula pubescens, Johansson (1986) note par exemple une baisse de teneurs en amidon racinaire, $30 \mathrm{j}$ après la coupe. Chez l'ailante, au contraire, les coupes successives provoquent une augmentation de l'amidon des racines latérales. La souche est caractérisée par une hydrolyse importante de l'amidon. Mais à la différence des observations de Dubroca et Saugier (1988) qui mettent en évidence un affaiblissement considérable du niveau des réserves de la souche de châtaignier recépée 2 fois dans l'année, la souche d'ailante reste un compartiment très riche en glucides solubles, même après 3 coupes successives. La souche ne peut donc pas être considérée comme un simple support mécanique du système brin-racine tel que l'ont décrit chez le chêne-liège Destremeau et Roderbourg (1968).

II ne faut pas oublier que les pousses produites par le recépage sont d'une manière générale nettement plus productrices en terme de biomasse, comparées aux germinations de même âge (Anderson et Zsuffa, 1975). Le recépage a pour conséquence le développement d'un système photosynthétique jeune, plus efficace visà-vis de la photosynthèse. De plus, la coupe diminue l'éloignement entre les feuilles et les racines, et selon Kazarjan (1969) plus le feuillage est éloigné, moins la mise en réserve des métabolites est efficace. L'augmentation des réserves glucidiques sous l'effet des coupes et dans l'ensemble de la plante traduit chez l'ailante une grande efficacité photosynthétique soulignée par Marek (1988).
L'ablation caulinaire peut entraîner un changement dans la localisation des zones de stockage des métabolites de réserves. Ainsi, chez le platane, Clair-Maczulajtys et Bory (1988) ont montré que certaines pratiques d'élagage provoquent un changement dans la répartition des réserves glucidiques du houppier. La modification du compartimentage des réserves est, chez l'Ailanthus glandulosa, un des effets persistants du recépage. La mise en réserve des glucides se fait préférentiellement dans les racines latérales et ce au détriment du pivot. Ceci serait peut-être dû à l'établissement d'une circulation privilégiée des sèves entre les racines latérales et le brin. Plusieurs auteurs ont observé qu'ii existe, au niveau d'une souche, une alimentation privilégiée des brins par les racines situées à proximité. C'est le cas chez le bouleau (Bedeneau et Pagès, 1984) ou le châtaignier (Aymard et Fredon, 1986). La souche devient, après 3 recépages successifs, la zone principale d'accumulation de sucres solubles. Au contraire, le collet, chez le plant non recepé, demeure très pauvre en glucides métabolisables pendant toute la durée de l'expérience et est même dépourvu d'amidon lors de la rentrée en serre des jeunes plants. Cette caractéristique a déjà été décrite chez des arbres d'une quinzaine d'années où la base des troncs présente des teneurs extrêmement faibles en amidon (Bory, 1983).

Chez les jeunes plants d'ailante, ClairMaczulajtys (1984) a mis en évidence que les basses températures et la salinité provoquent, comme les recépages successifs, une augmentation des lipides totaux dans le système souterrain. D'une manière générale, chez bon nombre de végétaux ligneux, le froid est responsable de l'accumulation de lipides (Zimmermann, 1971; Tschager et al, 1982) et cela s'accompagne souvent d'une élévation du degré d'insaturation des acides gras totaux. II y a 
donc là une similitude avec les effets des recépages successifs.

La coupe, en favorisant, d'une part le développement des racines latérales porteuses de bourgeons racinaires, et d'autre part l'accumulation de réserves à ce niveau, permet au jeune plant une meilleure adaptation à la colonisation des sols nus. Comme le soulignent Trabaud et Méthy (1988), pour des chênes sempervirents, les réserves du système racinaire jouent un rôle essentiel dans les premiers stades de la reconstitution des parties aériennes détruites. C'est également une des explications des capacités de régénération de l'ailante après le passage des incendies (Clair-Maczulajtys, 1984). Cette étude tend à montrer que l'ailante glanduleux est une des rares espèces ligneuses non seulement capable de supporter des rotations annuelles mais aussi d'avoir un système racinaire dont la croissance est renforcée par cette pratique. Son utilisation potentielle dans la création de zones pare-feux ou comme fixateur de sols devrait être sérieusement envisagée en France.

\section{RÉFÉRENCES}

Abusrewill GS, Larsen FE, Fritts R (1983) Prestorage and post-storage starch levels in chemically and hand. J Am Soc Hort Sci $108,20-23$

Anderson HW, Zsuffa L (1975) Yield and wood quality of hybrid cottonwood growing in twoyear rotation. Ont Min Nat Res For Res Rep 101, 1-35

Ashwell G (1957) Methods in Enzymology, 3, Acad Press, New York, 73

Aymard M, Fredon JJ (1986) Étude des relations entre une racine et les rejets de la souche chez Castanea sativa Mill. Ann Sci For 43, 351-364

Bamber RK, Humphreys FR (1965) Variation in sapwood starch levels in some australian forest species. Austr For 29, 15-23

Bedeneau M, Pagès L (1984) Répartition de la sève entre les jeunes rejets de bouleau étu- diés à l'aide d'un phytocide. Ann Sci For 41, 131-141

Bory G (1983) Quelques aspects de la biologie de l'Ailanthus altissima (Mill) Swingle. Mouvements de métabolites, croissance, développement, sécrétion et floraison chez divers types d'arbres. Thèse doctorat d'État, université Paris 7, $287 p$

Carreau JP, Dubacq JP (1978) Adaptation of a macro-scale method to the micro-scale for fatty acid methyl transesterification of biological lipid extracts. J Chromatogr 151, 384-390

Caruso JL (1974) In vitro bud formation in stem segments of Ailanthus altissima. New Phytol $73,441-443$

Clair-Maczulajtys D (1984) Quelques aspects de la biologie de l'Ailanthus altissima (Mill) Swingle. Étude de la double stratégie de reproduction par graines et par drageonnement en relation avec les métabolites de réserve. Thèse doctorat d'État, université Paris 7, $441 p$

Clair-Maczulajtys D, Bory G (1983) Observations sur les potentialités organogènes chez l'Ailanthus glandulosa Desf (Simarubacées). Bull Soc Bot Fr 130, 103

Clair-Maczulajtys D, Bory G (1985) Évolution de la composition lipidique des cataphylles chez l'Ailanthus glandulosa au cours de l'ouverture du bourgeon. Physiol Vég 23, 275-282

Clair-Maczulajtys D, Bory G (1988) Modifications de la répartition des glucides de réserve sous l'effet de l'élagage, chez deux arbres d'ornement (Platanus acerifolia Willd et Tilia platyphyllos Scop). Bull Soc Bot Fr 135, 4153

Destremeau D, Roderbourg J (1968) Éléments pour l'étude du traitement en taillis de la forêt de chêne-liège de Mamora. Répartition de la sève entre les rejets. Ann Rech For Maroc 2, 237-242

Dubroca E, Bory G (1981) Composés glucidiques et azotés et résistance à la sécheresse chez l'Ailanthus altissima. Biochem Syst Ecol 9, 283-288

Dubroca E, Saugier B (1988) Effet de la coupe sur l'évolution saisonnière des réserves glucidiques dans un taillis de Châtaigniers. Bull Soc Bot Fr 135, 55-64

Eliasson L (1971) Growth regulators in Populus tremula III. Variation of auxin and inhibitor 
level in roots in relation to root sucker formation. Physiol Plant 25, 118-121

Johansson $T$ (1986) Development of suckers by two-year-old birch (Betula pendula Roth) at different temperatures and light intensities. Scand J For Res 1, 17-26

Kauppi A, Rinne P, Ferm A (1988) Sprouting ability and significance for coppicing of dormant buds on Betula pubescens Ehrh Stumps. Scand J For Res 3, 343-354

Kazarjan VO (1969) Le vieillissement des plantes supérieures. Navka, Moscou, Traduction française A Riedacker, CNRF, Nancy, $194 p$

Kovacs M, Opauszky I, Klincsek PK, Podani J (1982) The leaves of city trees as accumulation indicators. In: Monitoring of air pollutants by plants. Methods and problems (LS Steubing, HJ Jager, eds) Dr W Junk, The Hague, 149-153

MacReady RM, Guggolz J, Silviera V, Owens HS (1950) Determination of starch and amylase in vegetables. Anal Chem 22, 11561158

Marek M (1988) Photosynthetic characteristics of Ailanthus leaves; Photosynthetica 22, 179183

Ohmoto T, Tanaka R, Nikaido T (1976) Studies on the constituents of Ailanthus altissima: on the alkaloidal constituents. Chem Pharm Bull (Tokyo) 24, 1532-1536

Parker J (1979) Effects of defoliation and root height above a water table on some red oak root metabolites. J Am Soc Hort Sci 104, 417-421

Reich L (1985) Carbohydrates in the apple tree : effects associated with pruning and deblossoming. Hortscience 20, 238-240

Rinne P, Kaupi A, Ferm A (1987) Induction of adventitous buds and sprouts on birch seed- lings (Betula pendula Roth and $B$ pubescens Ehrh). Can J For Res 17, 545-555

Schier GA (1973) Seasonal variation in sucker production from excised roots of Populus tremuloides and the role of endogenous auxin. Can J For Res 3, 459-461

Schier GA, Zasada JC (1973) Role of carbohydrate reserves in the development of root suckers in Populus tremuloides. Can J For Res 3, 243-250

Sussex IM, Clutter ME, Lutinski JB, Dilks LJ (1960) Extraction and biological properties of an antifungal fraction of woody plants. Bot Gaz 121, 171-175

Taylor JS, Blake TJ, Pharis RP (1982) The role of plant hormones and carbohydrates in the growth and survival of coppiced Eucalyptus seedlings. Physiol Plant 55, 421-430

Tew RK (1970) Root carbohydrate rèserves in vegetative reproduction of Aspen. For Sci 16, 318-320

Trabaud L, Methy M (1988) Modifications dans le système photosynthétique de repousses apparaissant après feu de deux espèces ligneuses dominantes des garrigues méditerranéennes. Acta CEcol CEcol Plant 9, 229-243

Tschager A, Hilcher H, Franz S, Kull U, Larcher W (1982) Jahreszeitliche dynamik der fettspeicherung von Loiseleuria procumbens und anderen Ericaceen der alpinen Zwergstrauchheide. Acta $C_{E c o l} C_{E c o l}$ Plant 3, 119134

Vogt AR, Cox GS (1970) Evidence for the hormonal control stump sprouting by oak. For Sci $16,165-171$

Zimmermann MH (1971) Storage, mobilization and circulation of assimilates. In: Trees: Structure and Function (MH Zimmermann, CL Brown, eds), Springer Verlag, Berlin, 307-321 